\title{
BMJ Open Improving the network management of integrated primary mental healthcare for older people in a rural Australian region: protocol for a mixed methods case study
}

\author{
Jeffrey Fuller, ${ }^{1}$ Candice Oster, ${ }^{1}$ Suzanne Dawson, ${ }^{1}$ Deb O'Kane, ${ }^{1}$ Sharon Lawn, ${ }^{2}$ \\ Julie Henderson, ${ }^{1}$ Adam Gerace, ${ }^{1}$ Richard Reed, ${ }^{2}$ Ann Nosworthy, ${ }^{3}$ Philip Galley, ${ }^{4}$ \\ Ruth McPhail, ${ }^{5}$ Eimear Muir Cochrane ${ }^{1}$
}

To cite: Fuller J, Oster C, Dawson S, et al. Improving the network management of integrated primary mental healthcare for older people in a rural Australian region: protocol for a mixed methods case study. BMJ Open 2014;4:e006304. doi:10.1136/bmjopen-2014006304

- Prepublication history and additional material is available. To view please visit the journal (http://dx.doi.org/ 10.1136/bmjopen-2014006304).

Received 5 August 2014 Accepted 18 August 2014

\section{CrossMark}

For numbered affiliations see end of article.

Correspondence to Professor Jeffrey Fuller; jeffrey.fuller@filnders.edu.au

\section{ABSTRACT}

Introduction: An integrated approach to the mental healthcare of older people is advocated across health, aged care and social care sectors. It is not clear, however, how the management of integrated servicing should occur, although interorganisational relations theory suggests a reflective network approach using evaluation feedback. This research will test a network management approach to help regional primary healthcare organisations improve mental health service integration.

Methods and analysis: This mixed methods case study in rural South Australia will test facilitated reflection within a network of health and social care services to determine if this leads to improved integration. Engagement of services will occur through a governance group and a series of three 1-day service stakeholder workshops. Facilitated reflection and evaluation feedback will use information from a review of health sector and local operational policies, a network survey about current service links, gaps and enablers and interviews with older people and their carers about their help seeking journeys. Quantitative and qualitative analysis will describe the policy enablers and explore the current and ideal links between services. The facilitated reflection will be developed to maximise engagement of senior management in the governance group and the service staff at the operational level in the workshops. Benefit will be assessed through indicators of improved service coordination, collective ownership of service problems, strengthened partnerships, agreed local protocols and the use of feedback for accountability.

Ethics, benefits and dissemination: Ethics approval will deal with the sensitivities of organisational network research where data anonymity is not preserved. The benefit will be the tested utility of a facilitated reflective process for a network of health and social care services to manage linked primary mental healthcare for older people in a rural region. Dissemination will make use of the sectoral networks of the governance group.

\section{Strengths and limitations of this study}

- Testing of a theoretically based planning framework for an informal health service network across health and social care in the complex field of older people's mental health.

- Engagement of health service stakeholder organisations as participants in the study design, conduct and use of findings.

- Use and dissemination of findings optimised by the participation of service stakeholders.

- Potential for limited transferability due to differing capacity and context in other health service networks.

- Potential for harm in organisational case studies using participatory methods where there are risks to anonymity and confidentiality.

\section{INTRODUCTION}

Population ageing is common worldwide and in Australia the population aged 65 years and above is projected to increase rapidly in total numbers and in the proportion of the population. ${ }^{1}$ While the majority of older people have good mental health, a large number will experience mental health problems including diagnosed mental illness. It is reported that mental illness affects up to $50 \%$ of older people living in residential aged care, up to $48 \%$ in hospital settings and $20 \%$ in community dwellings. ${ }^{2}$ Furthermore, many individuals above 65 years will experience multiple chronic mental and physical health conditions, making healthcare delivery more complex. ${ }^{3}$

In Australia $36 \%$ of the population aged 65 and above live outside major cities, with reported poorer health outcomes and unmet 
needs for mental healthcare. ${ }^{2} 5$ People aged 65 years and above are less likely to access general practitioner mental health services and mental health professionals. ${ }^{6}{ }^{7}$ Rural older people are particularly disadvantaged by a lack of available local mental health professionals; they often rely on visiting psychiatrists and can travel large distances to access specialist mental health services. ${ }^{2} 8$ The consequences of lack of adequate primary mental healthcare for older people includes frequent and longer acute hospitalisation, deterioration of physical and mental health, as well earlier admission to residential care. ${ }^{9} 10$

An older person with a mental health problem may require input from health, aged care and social care services, and so there is a need for a well networked range of local services, especially in rural locations. Owing to the complexity of health problems experienced by older people, there is widespread support internationally for the provision of a more integrated approach to care, with a growing evidence base for the effectiveness of this. ${ }^{11-15}$ Benefits of a more integrated approach to care include: better outcomes for the client and carer; improved access to and experience of services; and better use of existing resources. ${ }^{16}$

Governments and professional groups have prioritised mental health service delivery based in primary care with the need for integration and the provision of the 'right service' at the 'right time', which includes transition between various service sectors. ${ }^{6}{ }^{17-20}$ To address the need for more integrated services through planning, networking and coordination, the Australian Government established a model of regional primary healthcare organisations, called Medicare Locals. ${ }^{i}$ State Governments in Australia have also developed Local Health Networks to manage the delivery of public hospital as well as some out-of-hospital services. Together, Medicare Locals and Local Health Networks, along with a range of general practice, aged care and social care organisations, plan and provide care for older people with mental health problems.

Despite support for integrated care it is not often clear in policies what this means specifically or what is needed to effectively integrate services. In part this problem relates to the use of the term 'integration' without specifying its interpretation, which could cover a range of levels from the total integration of organisations or functions under one umbrella through to the formation of loose networks or alliances. ${ }^{21}$ In addition, the management of integrated health servicing across numerous organisations requires different management dynamics than for traditional bureaucratic and marketbased forms of healthcare organisation, because

\footnotetext{
í Since beginning this study a change in the national government has seen the cancellation of the Medicare Local Program. At the time of writing the government has made a commitment to regional primary health care organisations but has not determined their form or function.
}

organisational independence is retained but cooperation is required. A network approach, whereby a number of independent organisations cooperate to achieve mutual goals has been suggested; but to make change in a network a manager needs to engage in the ongoing negotiation of commitment to the network through facilitating the development of trust and reciprocity between partners. ${ }^{22} \mathrm{~A}$ recent review examining the effectiveness of links in primary mental healthcare identified a paucity of real world testing of practical management models of integrated health servicing. ${ }^{23}$ This means that there are not clear and well-understood processes through which a network of organisations can work together to meet the mental healthcare needs of older people.

\section{METHODS AND ANALYSIS}

In the current Australian context of Medicare Locals and Local Health Networks, the aim of this study is to test a framework for planning and management of a network of services in the provision of primary mental healthcare for older people in a rural area. The research was funded in October 2013 through a nationally competitive grant from the Australian Primary Health Care Research Institute. ${ }^{24}$ Data collection started in the field in February 2014 with analysis to be completed in March 2015.

\section{Framework for network planning using facilitated reflection}

The network planning approach using facilitated reflection is based on inter-organisational relations theory. Hibbert et $a 2^{25}$ make the point that because collaborative relationships are idiosyncratic, then the generalisable feature of network management is the development of 'handles for reflective practice' (p.405) in which partners formulate their actions in light of their own circumstances and competencies. From this starting point of reflective practice we drew from a previous narrative review of the first author on the linkage strategies and enablers in primary mental healthcare. Ten linkage strategies were identified ${ }^{11}$ as well as the factors that enable the development of these strategies. ${ }^{23}$ Table 1 describes the linkage strategies and figure 1 shows the enablers. The process that brings the enablers together is evaluation feedback (reflective practice), which in this proposed study will occur through facilitated reflection. We will compare this framework to what we find in this case study.

The facilitated reflection involves the collective use of feedback on data obtained from a network survey, service provider interviews and help seeking journeys described by clients or their carer. This reflection will be facilitated to enable problem solving in three 1-day workshops with service stakeholders. The rationale is that network management must make use of collective reflection more so than in a bureaucracy or a market-based arrangement because leadership and authority in a 
Table 1 Linkage strategies in primary mental health care

\begin{tabular}{|c|c|c|}
\hline Category & Strategy & Description \\
\hline \multirow[t]{4}{*}{$\begin{array}{l}\text { Direct collaborative } \\
\text { activities }\end{array}$} & Link working & $\begin{array}{l}\text { Organisational tasks connecting } 2+\text { services-may involve limited clinical } \\
\text { intervention but not expert clinical advice or structured liaison }\end{array}$ \\
\hline & Co-location & $\begin{array}{l}\text { Face to face-location of mental health worker in primary care-must } \\
\text { provide treatment in primary care }\end{array}$ \\
\hline & Consultation liaison & $\begin{array}{l}\text { Person } 1 \text { provides advice about care to person } 2 \text { without transfer of care } \\
\text { — can be via a link worker }\end{array}$ \\
\hline & Care management & $\begin{array}{l}\text { Coordination of care-including assessment, review, follow-up and care } \\
\text { management plan; linking with other services or defined care pathway }\end{array}$ \\
\hline \multirow[t]{2}{*}{ Agreed guidelines } & Protocols & Agreed treatment algorithm—pharmacotherapy or psychological therapy \\
\hline & Stepped care & Treatment escalation or de-escalation procedure to other providers \\
\hline \multirow[t]{3}{*}{$\begin{array}{l}\text { Communication } \\
\text { systems }\end{array}$} & $\begin{array}{l}\text { Enhanced } \\
\text { communication }\end{array}$ & Formal process with feedback—meetings, shared medical record \\
\hline & Enhanced referral & Expedited access-explicit referral criteria/ process. \\
\hline & $\begin{array}{l}\text { Electronic } \\
\text { communication system }\end{array}$ & Tele-video conference connecting 2+ services \\
\hline Service agreement & Service agreement & $\begin{array}{l}\text { Memorandum of understanding-formal contract/ funding mechanism } \\
\text { about how services work together }\end{array}$ \\
\hline
\end{tabular}

network are more distributed among services. ${ }^{22} 25 \quad 26$ Sheaff $e t a l^{27}$ describe this reflective use of feedback as the 'evidence basing' incentive that managers can use to motivate participation in a network, to show members what is working and the outcomes that are being achieved. ${ }^{28}$ Hence, our assumption for testing is that the implementation of change in a network will be driven by the collective reflection on feedback about the network. The extent to which information feedback is used by the services will be influenced by their level of commitment to working together (conducive context) and that a conducive context will be created if there are linkage enablers. ${ }^{29-31}$ The research design to promote collective reflection is shown in figure 2 as the framework for network planning.

\section{Research questions}

The study asks the following five questions:

1. What organisational links currently exist in a rural region for the provision of mental healthcare for older people?

2. What are the gaps, barriers and enablers in linking services, as perceived by key service stakeholders, older people and their carers?

3. What is the role of policy in supporting the development of linked care for older people in a rural region?

4. What links could be established between services for the mental healthcare of older people?

5. How does the use of facilitated reflection enable an informal network to plan and manage linked primary mental healthcare for older people in a rural region?

\section{Setting}

The research is a case study located in the southern part of the Adelaide Hills, Fleurieu and Kangaroo Island rural region in South Australia. This region has a growing older population with a $52 \%$ increase in those aged 65 years and above from 2001 to $2011^{32}$ The region is typical of many Australian rural locations that are within $100 \mathrm{~km}$ of metropolitan centres, but which still face difficulties in service access, coordination and follow-up because of differences in funding criteria and boundaries between services. While there is no purposive or managed network of services related to older people's mental health in the region, there are a number of regular interagency meetings and there is a 'Positive Ageing Taskforce' involving a range of service providers that is managed by the local government and funded by the national government. There is no binding commitment made by local services to the Taskforce and it has no formal decision-making function or specific resources. Hence, the network of services is best described as loose, informal and not explicitly defined. Specialist mental health services are delivered to older people living in the region by the local communitybased mental health team with a consultation liaison service available at the large mental health hospital some $80 \mathrm{kms}$ away in the capital city. Acute inpatient units providing psychiatric inpatient treatment for persons 65 years and above are also located in the capital city, but these are run by metropolitan networks of the South Australian Government health system.

At the request of the Positive Ageing Taskforce we recently undertook a study in this region exploring the views of health and social care providers about the barriers to mental healthcare for older people. ${ }^{33}$ We found a wide range of organisations provide care to older people and that while collaborative arrangements tended to be informal, these arrangements were well regarded. Barriers to more integrated services related to the difficulty in getting all relevant agencies to become involved in these collaborative arrangements, insufficient knowledge about available services, inadequate referral 
Figure 1 Linkage enablers in primary mental healthcare.

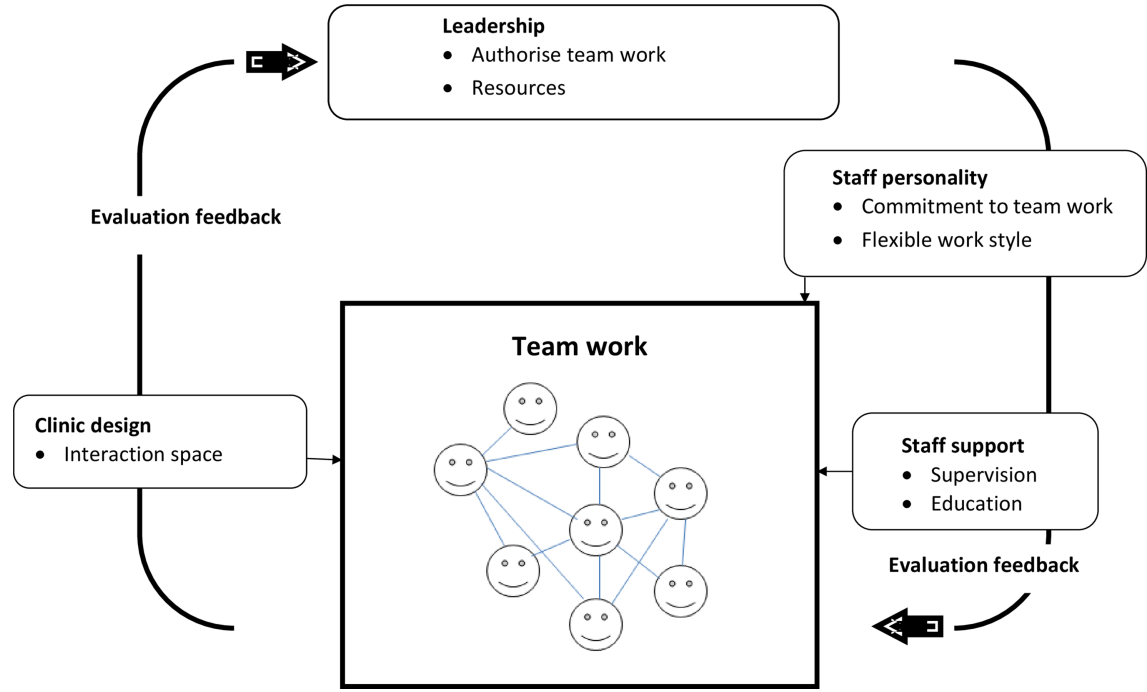

processes, competition for funding and also gatekeeping that restricts which clients a service will accept. The lack of attention to the client's physical and mental health issues by services was thought to be a problem.

\section{Participants}

A governance group will be established comprising three to five key senior staff with management responsibilities in rural mental health, regional primary healthcare planning and social care related to older persons' mental health in the region. This will be a small decision-making group that will also include the project investigators. The group will meet throughout the project to establish research governance and inform network planning. Meetings will occur before and following each of three service stakeholder workshops and also at the conclusion of the project in order to review the effectiveness of the framework for network planning for broader application.

Up to 30 participants for service key informant interviews and stakeholder workshops will be recruited via purposive snowball sampling starting first from the governance group. We will identify organisations and key staff across mental health, primary care, aged care and social care services that provide mental health and related services to older people in the region.

Ten older people (aged 65 and older) and/or their carers who have sought mental healthcare in the region will be interviewed to explore their journeys to care. These participants will be purposively recruited with the assistance of the service providers in the key informant interviews and stakeholder workshops.
Figure 2 Framework for network planning.

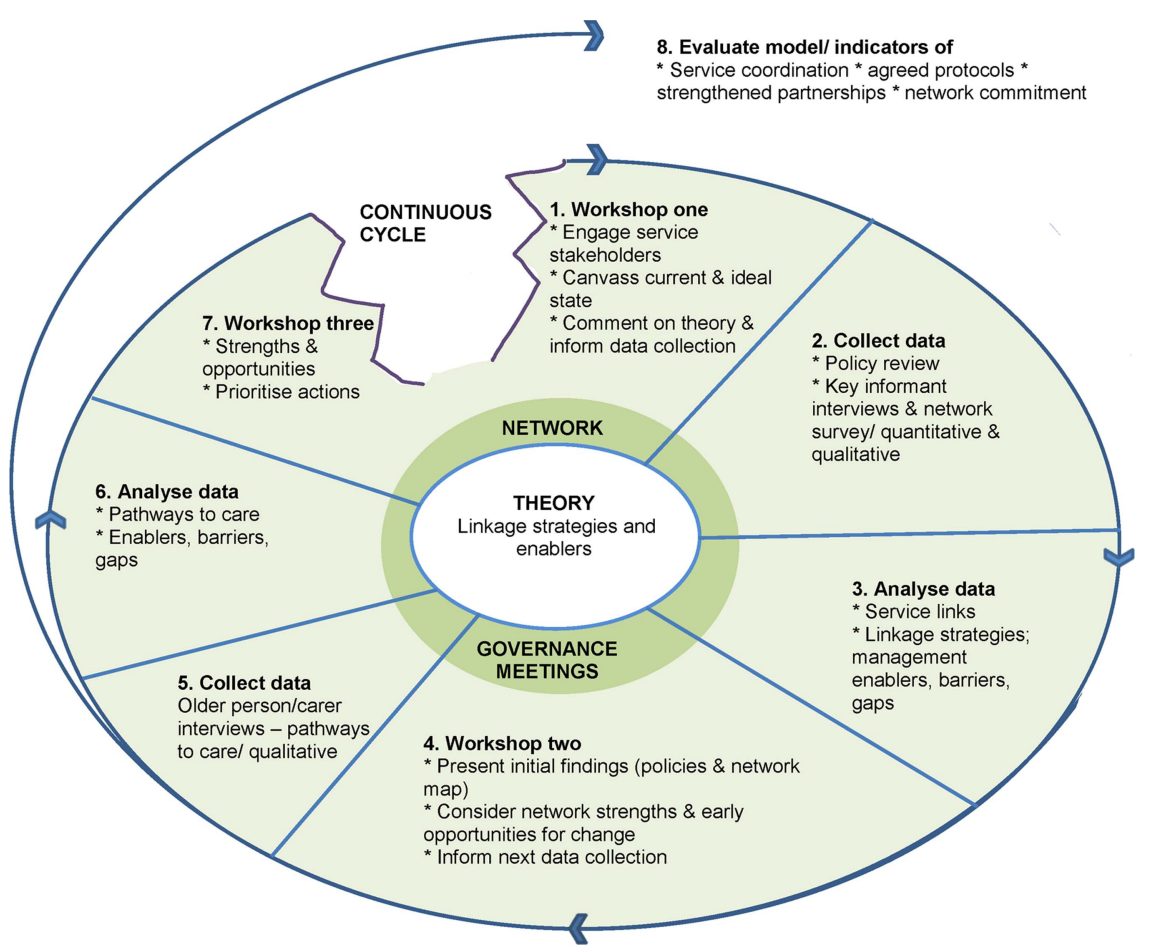


Table 2 Research questions and associated data collection and analysis methods

Question 1

What organisational links currently exist in a rural region for the provision of mental healthcare for older people?

\section{Question 2}

What are the gaps, barriers and enablers in linking services, as perceived by key service stakeholders, older people and their carers?

\section{Organisational network analysis (interview survey)}

Up to 30 key informants from relevant health and human service organisations (identified through the governance group) will be interviewed using organisational network analysis to explore the regional service network structure. Organisational network analysis, also known as social network analysis, gathers information about which organisations are linked, in this case on the activities of information sharing, referrals and the management, planning and operation of services for the mental healthcare of older people. $^{3435}$ This provides quantitative information specific to the local network and to the unique and complex interactions between these organisations. See online supplementary appendix 1 for the survey and interview guide.

Measures of organisational network analysis will be used to provide information about which organisations are linked, the number of links in the network, the types of interactions between organisations (eg, exchanging information, referrals and planning), and the level and strength of each relationship. ${ }^{34}$ Maps displaying the patterns of connections between organisations will be generated utilising the UCINET software. ${ }^{36}$ The visual and detailed nature of organisational network maps serves as a powerful heuristic device for discussion about the structure of the network, about which services are linked and on which activities. See online supplementary appendix 2 for a hypothetical map.

\section{Qualitative interviews: key informant service providers and care seeking journeys}

Qualitative interviews will explore the above 30 service key-informants perspectives on the linkage gaps, barriers and enablers between services.

In addition 10 older people and their carers will be interviewed using the Pathways Interview Schedule. ${ }^{37}$ This is a semistructured instrument designed for the systematic gathering of information on their routes to and sources of care. Two groups will be purposively selected with the assistance of the service key informants: those who have successfully negotiated a care journey and those who have not.

Framework analysis will be used to explore the present linkage strategies and management gaps, barriers and enablers between mental health, aged care, primary care and social care services, and how these affect people's help seeking experience and journey. Framework analysis is a qualitative method that is suited to applied research with specific questions, a limited timeframe, a predesigned sample, and a priori issues that are to be explored. ${ }^{38}$ The NVivo software package will be used to manage and assist analysis of the qualitative data. ${ }^{39}$

\section{Policy analysis}

Policy documents at the national, state and the local operational levels will be examined for their relevance to the development of integrated mental healthcare for older people in the region. The national and state level policies will be identified through online searches and advice from the governance group, while operational policies (eg, service plans, local guidelines and protocols etc.) will be identified though the service key informant interviews. The local operational policies from the various sectors (primary care, mental health, aged care, social care services) will be examined for congruence, direction and resource opportunities.
Findings from the project will be presented to the service stakeholders progressively throughout the project to facilitate improved service coordination. This will occur at management (governance group) and operations (stakeholder workshops) levels in order to maximise engagement and opportunities for change. 
Table 2 Continued

Question 4

What links could be established between services for the mental healthcare of older people?

\section{Facilitated network reflection}

Data from the methods above will be fed back in the governance group and in the three service stakeholder workshops held over 12 months. The purpose of each workshop is to progressively work towards the final goal of securing commitment among a group of stakeholder services for a network problem solving approach to the delivery of mental healthcare to older people in the region.

- Workshop 1: Comment on and adapt the network planning framework, identify potential champions and begin to explore organisational readiness and capacity for change. Data pertaining to participants' perceptions of current as well as ideal links will also be collected.

- Workshop 2: Findings related to the organisational network analysis, service key informant interviews and policy analysis will be fed back. This information will provide the participants with the opportunity to consider network strengths and weaknesses and explore early opportunities for change. After the organisational network analysis and key informant data are fed back, small group discussion will be guided by the following prompts: (1) Does the network look as you would expect on the maps/ what stands out/ what seems missing? (2) What else do you want to know -the maps show the links, not what goes down them? (3) Do the maps suggest what might be working and what else might be needed?

- Workshop 3: Final analysis will be added to that presented at workshop 2 and will include the data on older people's care seeking journeys. The current state of the network will be examined against the theory on linkage strategies and enablers to identify strengths, weaknesses and opportunities for change. From this examination the priorities for action will be proposed to the governance group for improved integration in regional mental health servicing for older people.

Workshop discussions will be recorded and used along with other data in the case study analysis to answer question 5 .

\section{Case study}

Data will be collected throughout the study to evaluate the utility of the 'framework for network planning' for an informal network to develop more integrated mental healthcare for older people in this rural region. These data will include records of the governance group and service stakeholder workshops. In addition, further service key informant interviews will be conducted at the end of the project to explicitly explore whether they have found the framework useful or not using the following prompts:

1. Can you describe how you found the facilitated reflective process (workshops, survey, interviews) as a way to manage the range of services as a network, in terms of the following:

- Acceptability of the process

- Ability to identify and act on issues

2. Did the project achieve what you expected?

3. Would you use this process again-would you change anything? Engagement at senior management (governance group) and operational (key stakeholder) levels will be essential to develop the trust and insight to accurately assess the applicability of the network planning framework.

\section{Data collection and analysis}

The following table 2 shows the five research questions and the methods that will be used to collect and analyse the data:

\section{ETHICS, BENEFIT AND DISSEMINATION}

The ethical risk to key informants from health and human service organisations is likely to be minimal; however, a risk to organisations relates to the display of their links with other organisations identified on network maps. Ethical guidelines for the use of network analysis within organisations have been developed covering fully informed consent and negotiated governance of research processes. ${ }^{40}$ While the data will be de-identified in public documents there is a potential for embarrassment during the workshops about the mapped position of organisations in the network as being prominent or not. To manage such risk, the governance group will provide advice and guidance 
throughout the project and manage sensitivities that may arise.

The research will benefit participating services through describing the existence and frequency of service links, which can then be used to improve links as needed. The main outcome will be a tested planning framework that can be used in an informal network to engage rural health and social care services in the improvement of service coordination for older persons' mental healthcare. If effective, the model should achieve the following outcomes:

- Better coordination of clinical and other supports to meet the mental health needs of older people.

- Collective ownership of innovative older persons' mental health service solutions.

- Strengthened partnerships between mental health, primary care, aged care and community support services.

- Improved and agreed referral pathways and local protocols between services.

- Evaluation feedback and accountability processes.

The manner in which these outcomes can be achieved in other regions will differ according to local contexts; however, the transferable outcome will be the network framework for integrated planning. The research team will develop recommendations for the application of the framework that will be fed up at a national level through peak bodies such as the National Primary Health Care Partnership, the Mental Health Council of Australia and the National Mental Health Consumer and Carer Forum. The involvement of senior staff from the main service stakeholders (the Medicare Local, the Positive Ageing Taskforce and the Country Health SA Local Health Network) will facilitate the implementation of the findings locally and more broadly across respective jurisdictions in mental health, primary care, aged care and community services.

The findings of the research will be produced using the Australian Primary Health Care Research Institute 1:3:25 format to policy makers for research reports.

\section{Author affiliations}

${ }^{1}$ School of Nursing \& Midwifery, Flinders University, Adelaide, Australia

${ }^{2}$ School of Medicine, Flinders University, Adelaide, Australia

${ }^{3}$ Southern Fleurieu \& Kangaroo Island Positive Ageing Taskforce, Victor Harbor, Australia

${ }^{4}$ Southern Adelaide-Fleurieu-Kangaroo Island Medicare Local, Bedford Park, Australia

${ }^{5}$ Country Health South Australia Local Health Network Mental Health Services, Adelaide, Australia

Contributors JF, CO and EMC designed the study. DOK, JH, SL, JH, AG and RR provided methodological input to the study design. AN, $P G$ and $R M$ provided input to the feasibility of the study and stakeholder engagement and data collection processes. CO and SD undertook the literature review. All authors contributed to the writing of the paper and all have approved the final version.

Funding The research reported in this paper is a project of the Australian Primary Health Care Research Institute, which is supported by a grant from the Commonwealth of Australia as represented by the Department of Health and Ageing. The information and opinions contained in it do not necessarily reflect the views or policy of the Australian Primary Health Care Research Institute or the Commonwealth of Australia (or the Department of Health and Ageing). Additional funding was also provided by a grant from the Nurses' Memorial Centre of South Australia and the Faculty of Medicine, Nursing and Health Sciences, Flinders University of South Australia.

Competing interests At the time of the study establishment AN, PG and RM were all employed in organisations that were a part of the service network that is the focus of this study.

Ethics approval Human Research Ethics Committees of the South Australian Health Department (HREC/13/SAH/126) and Flinders University (notification 10/2014).

Provenance and peer review Not commissioned; internally peer reviewed.

Data sharing statement The raw data are to be securely stored in a password protected server at the Flinders University of South Australia available to the research team only. In line with the participatory nature of the research design, analysed data in a de-identified form will be made available to participants. Hence these data along with the reporting to the funder will be available to other researchers on application to the corresponding author.

Open Access This is an Open Access article distributed in accordance with the Creative Commons Attribution Non Commercial (CC BY-NC 4.0) license, which permits others to distribute, remix, adapt, build upon this work noncommercially, and license their derivative works on different terms, provided the original work is properly cited and the use is non-commercial. See: http:// creativecommons.org/licenses/by-nc/4.0/

\section{REFERENCES}

1. Australian Bureau of Statistics. Population by Age and Sex Australian States and Territories. Ref: 3201.0. 2010.

2. Haralambous B, Xiaping L, Dow B, et al. Depression in older age: a scoping study. Australia: National Ageing Research Institute, 2009.

3. Glynn LG, Murphy AW, Smith SM, et al. Self-monitoring and other non-pharmacological interventions to improve the management of hypertension in primary care: a systematic review. $\mathrm{Br} J$ Gen Pract 2010;60:e476-88.

4. OECD. Health Reform Meeting the Challenge of Ageing and Multiple Morbidities. OECD Publishing, 2011. http://dx.doi.org/10.1787/ 9789264122314-en (accessed 28 Jul 2014).

5. Royal Australian \& New Zealand College of Psychiatrists. Priority must be given to investment that improves the mental health of older Australians. Position Statement 71. 2011. https://www.ranzcp.org/ Files/Resources/College_Statements/Position_Statements/ps71-pdf. aspx (accessed 28 Jul 2014).

6. Australian Government Department of Health. Fourth National Mental Health Plan: an agenda for collaborative government action in mental health 2009-2014. http://www.health.gov.au/internet/ publications/publishing.nsf/Content/mental-pubs-f-plan09-toc (accessed 28th Jul 2014).

7. Trollor JN, Anderson TM, Sachdev PS, et al. Prevalence of mental disorders in the elderly: the Australian National Mental Health and Well-Being Survey. Am J Geriatr Psychiatry 2007;15:455-66.

8. Davis $\mathrm{S}$, Bartlett $\mathrm{H}$. Healthy ageing in rural Australia: issues and challenges. Australas J Ageing 2008;27:56-60.

9. Draper B, Low L. What is the effectiveness of old-age mental health services? Copenhagen: WHO Regional Office for Europe, 2004. Health Evidence Network report http://www.euro.who.int/_data/ assets/pdf_file/0008/74690/E83685.pdf (accessed 28 Jul 2014).

10. Ryan AA, McCann S, McKenna H. Impact of community care in enabling older people with complex needs to remain at home. Int $J$ Older People Nurs 2009;4:22-32.

11. Fuller JD, Perkins D, Parker S, et al. Effectiveness of service linkages in primary mental health care: a narrative review part 1. BMC Health Serv Res 2011;11:72.

12. Kelly BJ, Perkins DA, Fuller JD, et al. Shared care in mental illness: a rapid review to inform implementation. Int J Ment Health Syst 2011;5:31.

13. Oliver D, Foot C, Humphries R. Making our health and care systems fit for an ageing population. London: Kings Fund, 2014.

14. Billings $\mathrm{J}$, Leichsenring $\mathrm{H}$, eds. Integrated health and social services for older persons: evidence from nine European countries. Aldershot: Ashgate, 2005.

15. Parekh A, Goodman R, Gordon C, et al. Managing multiple chronic conditions: a strategic framework for improving health outcomes and quality of life. Public Health Rep 2011;126:460-71. 
16. Humphries R, Curry N. Integrating health and social care. London: Kings Fund, 2011.

17. Joint Commissioning Panel for Mental Health. Guidance for commissioners of primary mental health care services. United Kindom, 2012. http://www.jcpmh.info/good-services/ primary-mental-health-services/ (accessed 28 Jul 2014).

18. World Health Organization and Wonca. Integrating Mental Health into Primary Care: a global perspective. Geneva: World Health Organization, 2008.

19. Commonwealth of Australia. Building a 21st century primary health care system: Australia's first national primary health care strategy. Australia: Commonwealth of Australia, 2010.

20. SA Health. Health Policy for Older People 2010-2016. Adelaide: Government of South Australia, 2010.

21. Ham C, Walsh N. Making integrated care happen at scale and pace. London: King's Fund, 2013.

22. Fuller J, Hermeston W, Passey M, et al. Acceptability of participatory social network analysis for problem-solving in Australian Aboriginal health service partnerships. BMC Health Serv Res 2012;12:152.

23. Fuller JD, Perkins D, Parker S, et al. Building effective service linkages in primary mental health care: a narrative review part 2. BMC Health Serv Res 2011;11:66.

24. Australian Primary Health Care Research Institute. The relationship between sub-acute care and primary health care. http://aphcri.anu. edu.au/research/network-research/research-programs/ relationship-between-sub-acute-care-and-primary-health (accessed 28 Jul 2014).

25. Hibbert $P$, Huxman $C$, Smith Ring P. Managing collaborative inter-organizational relations. In: Cropper S, Ebers M, Huxman C, et al, eds. The Oxford handbook of inter-organizational relations. Oxford University Press, 2008:390-416.

26. Johnston S, Green M, Thille P, et al. Performance feedback: an exploratory study to examine the acceptability and impact for interdisciplinary primary care teams. BMC Fam Pract 2011;12:14.

27. Sheaff R, Schofield J, Charles N, et al. The management and effectiveness of professional and clinical networks. Final Report. United Kingdom: National Institute for Health Research Service Delivery and Organisation Programme, 2011.
28. Blasinsky M, Goldman H, Unutzer J. Project IMPACT: a report on barriers and facilitators to sustainability. Adm Policy Ment Health 2006;33:718-29.

29. Helfrich CD, Damschroder LJ, Hagedorn HJ, et al. A critical synthesis of literature on the promoting action on research implementation in health services (PARIHS) framework. Implement Sci 2010;5:82.

30. Rycroft-Malone J, Kitson A, Harvey G, et al. Ingredients for change: revisiting a conceptual framework. Qual Saf Health Care 2002;11:174-80.

31. Conklin J, Kothari A, Stolee P, et al. Knowledge-to-action processes in SHRTN collaborative communities of practice: a study protocol. Implement Sci 2011;6:12.

32. Australian Government. My Region. Adelaide Hills, Fleurieu and Kangaroo Island. http://myregion.gov.au/profile/ adelaide-hills-fleurieu-and-kangaroo-island/data/population/age (accessed 28 Jul 2014).

33. Muir Cochrane E, O'Kane D, Barkway P, et al. Service provision for older people with mental health problems in a rural area of Australia Ageing Ment Health 2014;18:759-66.

34. Fried BJ, Johnsen MC, Starrett BE, et al. An empirical assessment of rural community support networks for individuals with severe mental disorders. Community Ment Health $J$ 1998;34:39-56.

35. Provan KG, Veazie MA, Staten LK, et al. The use of network analysis to strengthen community partnerships. Public Adm Rev 2005;65:603-12.

36. Ucinet 6 for Windows: Software for Social Network Analysis [program]. Harvard, MA: Analytic Technologies, 2002.

37. Sartorius N, Janca A. Psychiatric assessment instruments developed by the World Health Organization. Soc Psychiatry Psychiatr Epidemiol 1996;31:55-69.

38. Strivastava A, Thomson S. Framework analysis: a qualitative methodology for applied policy research. J Admin Governance 2009;4:72-9.

39. NVIVO 10 for Windows [program]. Doncaster, Victoria, Australia

40. Borgatti SP, Molina J-L. Toward ethical guidelines for network research in organizations. Soc Netw 2005;27:107-17. 